\title{
Home and office blood pressure: time to look at the individual patient
}

\author{
Peter W. de Leeuw ${ }^{1}$
}

Received: 27 August 2020 / Revised: 29 August 2020 / Accepted: 30 August 2020 / Published online: 2 February 2021

(c) The Japanese Society of Hypertension 2021

More than three centuries have passed since the English reverend Stephen Hales (1677-1761) and his student William Stukeley (1687-1765) began to measure arterial and venous blood pressure in dogs. Not much is known about these experiments, but in 1714, Hales performed his classic blood pressure measurement in an old mare for which he is still famous today [1]. Obviously, this was a "casual" measurement, but at least he was able to show the pulsatile nature of blood pressure and to establish that peak values of blood pressure coincided with contractions of the heart. Since then, we have come to learn a great deal more about ways to measure blood pressure, the significance of elevated blood pressure and the mechanisms involved in the pathogenesis and pathophysiology of hypertension. However, despite the enormous increase in knowledge, there are still many unknowns even with respect to the optimal method to estimate a person's blood pressure.

Currently, the practicing clinician has three different options to obtain a measurement of someone's blood pressure: conventional measurements in the office, 24-h ambulatory blood pressure monitoring and home blood pressure monitoring (HBPM) by the patient himself or herself. As far as office measurements are concerned, one has the additional choice of using either a classic aneroid type of sphygmomanometer to obtain the blood pressure measurement with the auscultation method or an (semi)automatic, usually oscillometric, device. Although all these methods gauge different aspects of the blood pressure profile, to some extent, they correlate with each other. However, HBPM measurements generally yield lower values than office measurements, and in untreated hypertensive patients, the

Peter W. de Leeuw

p.deleeuw@maastrichtuniversity.nl

1 Dept of Medicine, Maastricht University Medical Center and Cardiovascular Research Institute Maastricht (CARIM), Maastricht, The Netherlands difference between the two tends to be somewhat larger in men than in women and to widen with age and increased blood pressure [2, 3]. In addition, HBPM-based treatment has been strongly recommended for controlling blood pressure [4].

In the latest issue of the Journal, Kadowaki and associates provide additional data on the difference between office and home blood pressure measurements [5]. These investigators took the data from a population sample of 1056 Japanese men who had participated in an epidemiological study on subclinical atherosclerosis, the SESSA study, and analyzed the differences between office and home blood pressure obtained in these men. Their data show that office and home pressures were, on average, comparable, albeit with a substantial standard deviation. Smoking increased the difference between home and office measurements in all participants, while a higher body mass index did so only in those who used antihypertensive drugs. On this basis, the authors conclude that particularly in smokers and obese men treated for hypertension, office pressures may underestimate home pressure, the pressure that is more relevant for prognosis.

While these results are certainly of relevance, there are also some caveats that we need to acknowledge. First, this study was carried out a long time ago, between 2006 and 2008 , and we cannot exclude the possibility that with the devices that are currently available, the results would have come out slightly differently. Indeed, the oscillometric devices that the investigators used in their study are no longer on the market. Moreover, as we can see on the dabl Educational Trust website (www.dableducational.org), the instrument that the investigators applied for measuring office pressure did not fulfill accepted validation criteria. Presently, there are very strict protocols that a device has to pass before it can be recommended for clinical use, and readers are referred to the website of the nonprofit organization STRIDE BP (www.stridebp.org), which was founded by hypertension experts, to determine which instruments are suitable for their purpose. It would also have been 
preferable if the group of Kadowaki had used the same measuring device for establishing office and home blood pressures.

Another point of interest is that office pressure was taken after the participant had been resting for 5 min while being unattended. Then, a nurse entered the room and took two measurements. Since the SPRINT study first published its results [6], there has been much debate about unattended versus attended blood pressure measurements and the risk of a white coat effect [7-9], but in the present study, this was probably of minor importance, if at all. Nevertheless, the protocol for both office and home measurements did not comply with the recommendations that we have today [10]. This also implies that we do not know precisely how reproducible the observed differences between the two pressure measurements were.

Notwithstanding the abovementioned criticisms, the paper by Kadowaki and colleagues reminds us of a problem that we often overlook in medical practice-we tend to look too much at averages when we are comparing, for instance, the results of two measurement techniques. This is perhaps the strongest point that they make. Because home blood pressure, on average, is lower than office pressure, we have set the limits of normalcy at a lower level for the former. However, interindividual differences may be substantial and may sometimes go in one direction and at other times in the opposite direction. Home blood pressure is, indeed, sometimes higher than office pressure, and perhaps there are reasons for that. In an era where we seem to be so enthusiastic about personalized medicine, we have to have an eye for such individual characteristics.

\section{Compliance with ethical standards}

Conflict of interest The author declares that he has no conflict of interest.
Publisher's note Springer Nature remains neutral with regard to jurisdictional claims in published maps and institutional affiliations.

\section{References}

1. Hall WD. Stephen Hales: theologian, botanist, physiologist, discoverer of hemodynamics. Clin Cardiol. 1987;10:487-9.

2. Verberk WJ, Kroon AA, Kessels AG, de Leeuw PW. Home blood pressure measurement a systematic review. J Am Coll Cardiol. 2005;46:743-51.

3. Niiranen TJ, Jula AM, Kantola IM, Reunanen A. Comparison of agreement between clinic and home-measured blood pressure in the Finnish population: the Finn-HOME Study. J Hypertens. 2006;24:1549-55.

4. Satoh M, Maeda T, Hoshide S, Ohkubo T. Is antihypertensive treatment based on home blood pressure recommended rather than that based on office blood pressure in adults with essential hypertension? (meta-analysis). Hypertens Res 2019;42:807-16. Author Correction. Hypertens Res. 2019;42:1231-3.

5. Kadowaki S, Kadowaki T, Hozawa A, Fujiyoshi A, Hisamatsu T, Satoh A, et al. Differences between home blood pressure and strictly measured office blood pressure and their determinants in Japanese men. Hypertens Res. 2021;44:80-87.

6. Group SR, Wright JT Jr., Williamson JD, Whelton PK, Snyder JK, Sink KM, et al. A randomized trial of intensive versus standard blood-pressure control. N. Engl J Med. 2015;373:2103-16.

7. Asayama K, Ohkubo T. Unattended automated measurements: office and out-of-office blood pressures affected by medical staff and environment. Hypertension 2019;74:1294-6.

8. Johnson KC, Whelton PK, Cushman WC, Cutler JA, Evans GW, Snyder JK, et al. Blood pressure measurement in SPRINT (systolic blood pressure intervention trial). Hypertension. 2018;71:848-57.

9. Asayama K, Ohkubo T, Rakugi H, Miyakawa M, Mori H, Katsuya $\mathrm{T}$, et al. Comparison of blood pressure values-self-measured at home, measured at an unattended office, and measured at a conventional attended office. Hypertens Res. 2019;42:1726-37.

10. Williams B, Mancia G, Spiering W, Agabiti Rosei E, Azizi M, Burnier M, et al. 2018 Practice Guidelines for the management of arterial hypertension of the European Society of Hypertension and the European Society of Cardiology: ESH/ESC Task Force for the Management of Arterial Hypertension. J Hypertens. 2018; 36:2284-309. 